\title{
EFECTOS DEL LODO RESIDUAL Y RESIDUOS ORGÁNICOS VERMICOPOSTEADOS SOBRE INDICADORES INORGÁNICOS Y CATALASA
}

\author{
Nancy SERRANO-GARCÍA ${ }^{1,2}$, Rocío VACA ${ }^{3}$, Jorge LUGO $^{3}$ y Pedro DEL AGUILA ${ }^{* 3}$
}

${ }^{1}$ Profesora de Universidad Tecnológica del Valle de Toluca, Carretera del Departamento del D.F. km 7.5, Santa María Atarasquillo, Lerma, México

${ }^{2}$ Egresada de Doctorado en Ciencias e Ingeniería. Universidad Autónoma de Baja California. Calle de la Normal S/N y Blvd. Benito Juárez Col. Insurgentes Este Mexicali B. C.

${ }^{3}$ Profesor investigador. Facultad de Ciencias UAEMex. Instituto Literario 100, CP. 50000. Toluca, México.

*Autor para correspondencia: delaguila.1959@gmail.com

(Recibido mayo 2015; aceptado noviembre 2015)

Palabras clave: lombriz de tierra, productos agronómicos, vermiestabilización

\section{RESUMEN}

En este trabajo se evaluaron indicadores inorgánicos y actividad enzimática de tres mezclas de vermicomposta hechas con estiércol bovino (EB), residuos domésticos (RD), lodo residual (LR) y suelo (Cambisol), para conocer su madurez. Los tratamientos fueron mezclas de estiércol de vaca y residuo doméstico a una relación 1:1 (T1), 3:1 (T2) y $100 \%$ de estiércol de vaca (T3); además, a todos los tratamientos se les agregaron el equivalente a $40 \mathrm{Mg} / \mathrm{ha}$ de LR. Los análisis de laboratorio se hicieron siguiendo las NOM-004 (2002) y 021 (2000) de SEMARNAT. Los resultados antes de hacer el vermicomposteo para RD, EB y LR indican que los metales pesados no rebasaron la norma NOM-004 (2002). La relación C/N fue apropiada y va de acuerdo con lo reportado por Yang et al. (2014). Para los tratamientos, la humedad fue adecuada (menor a $72 \%$ ) y el pH presentó un comportamiento ligeramente alcalino (7.93). El $\mathrm{C}_{\text {org }}$ para T2 y T3 presentaron mayor contenido (12.88 y $12.84 \%$ ) respectivamente, con respecto a T1 $(8.90 \%)$. El N total varió de 0.73 a $1.03 \%$ siendo el mayor valor para $\mathrm{T} 1$. La relación $\mathrm{C} / \mathrm{N}$ señala la estabilización de los tratamientos de acuerdo a la norma NMX-FF-109-SCFI (2007) siendo para T3 y T2 de 14.14 y 17.48 respectivamente y T1 de 8.78. La catalasa estuvo entre $0.61 \pm 0.15$ a $0.81 \pm 0.015 \mu \mathrm{mol} \mathrm{H}_{2} \mathrm{O}_{2} \mathrm{~g}^{-1} \mathrm{~h}^{-1}$ donde la mayor actividad se mostró en T3, con estos datos, se concluye que T1 se considera la mejor opción para uso agronómico.

Key words: earthworm, agronomic products, vermistabilization

\begin{abstract}
This study evaluated inorganic indicators and enzymatic activity of three mixtures of vermicompost made with cow manure (EB), domestic waste (RD), sewage sludge (LR) and soil (Cambisol), to know its maturity. Treatments were mixed with, cow manure and domestic waste in a relationship to 1: 1 (T1), 3: 1 (T2) and 100\% of cow manure (T3), in addition all treatments were added the equivalent of $40 \mathrm{Mg} / \mathrm{ha}$ of LR. Laboratory analyses were made following the NOM 004 and 021 of SEMARNAT. The results before making vermicomposting for RD, EB and LR indicate that heavy metals did not exceed
\end{abstract}


the NOM-004 (2002) norm. The $\mathrm{C} / \mathrm{N}$ ratio was appropriate indicate and is according to reported by Yang et al. (2014). For treatments it was adequate moisture (less than $72 \%$ ) and showed a slightly alkaline $\mathrm{pH}$ behavior (7.93). The $\mathrm{C}_{\text {org }}$ for $\mathrm{T} 2$ and $\mathrm{T} 3$ showed higher content (12.84 and $12.88 \%$ ) respectively, compared to T1 $(8.90 \%)$. Total $\mathrm{N}$ ranged from 0.73 and 1.03 being the most value for $\mathrm{T} 1$. The $\mathrm{C} / \mathrm{N}$ ratio indicates stabilization treatment according to the NMX-FF-109-SCFI (2007) norm, being to T3 and T2 respectively 14.14 and 17.48 and 8.78 for T1. Catalase was between $0.61 \pm 0.15$ to $0.81 \pm 0.015 \mu \mathrm{mol} \mathrm{H}_{2} \mathrm{O}_{2}$ $\mathrm{g}^{-1} \mathrm{~h}^{-1}$ where most activity was showed in T3, wit these data, we concluded that T1 is considered the best option for agriculture use.

\section{INTRODUCCIÓN}

Dentro del desarrollo sustentable, uno de los retos consiste en llevar a cabo un programa integral para el manejo de residuos sólidos orgánicos (RSO) que se generan tanto en ciudad como en campo, los residuos de tipo animal, vegetal y lodos residuales, presentan un problema ambiental al generarse grandes volúmenes de estos.

Los RSO son fuentes de materia orgánica (MO) y nutrientes que pueden ser empleados en beneficio de suelo y planta (Armijo et al. 2007). Una manera de reducir, reciclar y reutilizar los RSO es mediante el uso de tecnologías económicamente sustentables conocidas como composteo y vermicomposteo; el composteo consiste en la degradación de la MO que transita por una fase termofílica y favorece a la eliminación de patógenos; mientras que, el vermicomposteo presenta una etapa mesofílica y una bioxidación lo cual logra la estabilización de la MO promovida por la lombriz de tierra y microorganismos (Suthar 2009, Hernández-Rodríguez et al. 2012).

De los dos procesos anteriores se obtiene una $\mathrm{MO}$ estable que cumple con estándares para su uso como mejorador de suelo (Ngo et al. 2012). Para evaluar la estabilidad de la $\mathrm{MO}$ se consideran parámetros como $\mathrm{pH}$, conductividad eléctrica (CE), capacidad de intercambio catiónico $(\mathrm{CIC})$, carbono orgánico $\left(\mathrm{C}_{\mathrm{org}}\right)$, nitrógeno total, relación $\mathrm{C} / \mathrm{N}$ y actividad enzimática, entre otros (Campitelli y Ceppi 2008, Suthar 2009).

Específicamente la catalasa, se ha empleado como un indicador de la actividad microbiana y antioxidante durante el metabolismo aerobio y como un marcador en la actividad microbiana durante el proceso de la respiración aerobia (Ceron y Melgarejo 2005, Ouni et al. 2013). Por lo tanto, el objetivo en este trabajo fue evaluar la estabilidad de la vermicomposta utilizando diferentes mezclas de residuos orgánicos con la finalidad de obtener un producto para uso agronómico.

\section{MATERIALES Y MÉTODOS}

\section{Muestreo}

El muestreo del suelo (Cambisol) fue de tipo sistemático en un área de cultivo de $1000 \mathrm{~m}^{2}$ en el campus universitario "El Cerrillo" de la Universidad Autónoma del Estado de México. Se tomaron ocho muestras de $1500 \mathrm{~g}$ a $20 \mathrm{~cm}$ de profundidad en el horizonte Ap (generado por el arado). El lodo residual se obtuvo del filtro prensa de la planta de tratamiento de aguas residuales perteneciente a la empresa Operadora de Ecosistemas S.A. de C.V. y el estiércol bovino fue colectado de la posta de la Facultad de Medicina Veterinaria Zootécnica de la Universidad Autónoma del Estado de México (UAEMex).

Las muestras fueron secadas a la sombra y a temperatura ambiente y se homogeneizaron para obtener una muestra compuesta respectivamente. De la muestra compuesta, se separó una porción la cual se pasó por una malla de $2 \mathrm{~mm}$ para realizar la caracterización física y química y el resto se empleó para la elaboración del experimento. Los residuos orgánicos de tipo domésticos (verduras y cítricos) se trituraron mediante un mortero. La lombriz de tierra (Eisenia foetida) que se utilizó para el vermicomposteo se seleccionó considerando el indicador de madurez sexual (clitelo bien desarrollado) con un tamaño promedio de 4 a $6 \mathrm{~cm}$ y peso húmedo de $0.5 \mathrm{~g}$ por individuo (Garg et al. 2006).

\section{Diseño del Experimento}

Se trabajó con tres tratamientos en donde se adicionó estiércol bovino (EB), residuo doméstico (RD), lodo residual (LR) y suelo. El proceso de vermicomposteo se llevó a cabo por triplicado y se utilizaron recipientes de plástico de $20 \times 30 \times 20 \mathrm{~cm}$ (ancho x largo x altura) como tinas de compostaje de acuerdo a lo reportado por Pearson et al. (2000).

A los tres tratamientos se les agregó $500 \mathrm{~g}$ de suelo y el equivalente a $40 \mathrm{Mg} \mathrm{ha}^{-1}$ de LR (720 g) y 
13 lombrices de tierra ( $6.5 \mathrm{~g}$ ) y se llevó a una temperatura de $18 \pm 3^{\circ} \mathrm{C}$; la humedad se mantuvo a $75 \pm 5 \%$ durante todo el proceso (90 días).

Únicamente se varió la cantidad de estiércol bovino (EB) y residuo doméstico (RD) en los diferentes tratamientos, como se muestra a continuación:

$$
\begin{aligned}
& \mathrm{T} 1=\mathrm{EB}+\mathrm{RD} \text { relación } 1: 1(1500 \mathrm{~g}+1500 \mathrm{~g}) . \\
& \mathrm{T} 2=\mathrm{EB}+\mathrm{RD} \text { relación } 3: 1(2250 \mathrm{~g}+750 \mathrm{~g}) . \\
& \mathrm{T} 3=\mathrm{EB}(3000 \mathrm{~g}) .
\end{aligned}
$$

Todo esto se llevó a cabo por triplicado.

\section{Análisis de laboratorio}

Las muestras de suelo, EB y LR se realizaron por triplicado y fueron caracterizadas mediante los siguientes análisis: $\mathrm{pH}, \mathrm{MO}$ y $\mathrm{N}$ total a través de los métodos establecidos en la NOM-021-2000 (SEMARNAT 2000). La concentración total de $\mathrm{Cu}, \mathrm{Zn}$, $\mathrm{Ni} \mathrm{y} \mathrm{Pb}$ se determinó mediante espectrofotometría de absorción atómica (Perkin Elmer 3110), previa digestión ácida $\left(\mathrm{HNO}_{3}+\mathrm{HCl}\right.$ en una relación 4:1) en horno de microondas Mars 5 a través del método EPA 3050B.

A los 90 días después de iniciado el proceso de vermicomposteo se tomaron muestras de todos los tratamientos y se les determinó humedad por el método gravimétrico de acuerdo a la NOM-021-2000 (SEMARNAT 2000), pH en agua en una relación 1:2 (suelo: solución) mediante un potenciómetro Corning modelo 340.

La materia orgánica se determinó por el método de Nelson y Sonmers (1996). N total por el método de Kjeldahl (Bremner y Mulvaney 1982). La catalasa por el método de Trasar-Cepeda et al. (2003) midiendo el $\mathrm{H}_{2} \mathrm{O}_{2}$ residual a través de $\mathrm{KMnO}_{4}$.

\section{Análisis estadístico}

Para conocer la normalidad de los datos se graficaron, posteriormente se aplicó un análisis de varianza (ANOVA) y una prueba de Tukey para ver diferencias entre los tratamientos, con un $95 \%$ de confianza, con el paquete estadístico, STATGRAPHICS PLUS Versión 5.0 (Statiscal Graphics Corporation, 2000).

\section{RESULTADOS Y DISCUSIÓN}

\section{Suelo y residuos orgánicos}

En el cuadro I se muestran las características químicas del suelo, RD, EB y LR que se utilizaron en el experimento. El pH del suelo fue modernamente ácido de acuerdo con la Norma Mexicana NOM-004-2002 (SEMARNAT 2002), el RD mostró un $\mathrm{pH}$ neutro y concuerda con los estudios de Singh et al. (2005) que utilizaron residuos vegetales. El LR presentó valores neutros con base en la Norma Mexicana, NOM-021-2000 (SEMARNAT 2000) y el EB fue medianamente alcalino, al igual que en los estudios de Awasthi et al. (2015) que reportan un $\mathrm{pH}$ de $7.2 \pm 0.16$.

El contenido de MO fue bajo en el suelo de acuerdo a la norma NOM-004-2002 (SEMARNAT 2002), los RD y EB presentaron bajos contenidos de MO tal como lo reportaron Suthar y Sing (2008), no así el LR, que presentó un contenido de MO muy alto de acuerdo a la norma NOM-021-2000 (SEMARNAT 2000).

El contenido de $\mathrm{N}$ total para el suelo y los LR fue alto según las NOM-004 y NOM-021, respectivamente. Los otros dos residuos orgánicos se consideraron altos de acuerdo a los trabajos de Singh et al. (2005). La relación C/N para $\mathrm{RD}(16.8 \pm 1.2)$, suelo (14.0 \pm 1.2$)$, EB $(9.7 \pm 1.5)$ y LR $(7.4 \pm 1.1)$ donde este último se parece a

CUADRO I. CARACTERÍSTICAS QUÍMICAS DEL SUELO Y RESIDUOS ORGÁNICOS EMPLEADOS EN LA ELABORACIÓN DE LA VERMICOMPOSTA

\begin{tabular}{lcccc}
\hline Parámetro & Suelo & Residuo doméstico & Estiércol bovino & Lodo residual \\
\hline $\mathrm{pH}$ & $6.0 \pm 1.7$ & $6.8 \pm 0.3$ & $7.4 \pm 0.6$ & $7.1 \pm 0.4$ \\
$\mathrm{C}$ org $(\%)$ & $1.4 \pm 0.5$ & $18.5 \pm 0.2$ & $23.3 \pm 0.7$ & $30.4 \pm 0.4$ \\
$\mathrm{~N}(\%)$ & $0.1 \pm 1.9$ & $1.1 \pm 0.6$ & $2.4 \pm 0.8$ & $4.1 \pm 1.2$ \\
$\mathrm{C} / \mathrm{N}$ & $14.0 \pm 1.2$ & $16.81 \pm 1.2$ & $9.7 \pm 1.5$ & $7.4 \pm 1.1$ \\
$\mathrm{Cu}\left(\mathrm{mg} \mathrm{kg}^{-1}\right)$ & $175.0 \pm 5.7$ & $130.0 \pm 2.9$ & $235.0 \pm 6.8$ & $490.0 \pm 9.9$ \\
$\mathrm{Zn}\left(\mathrm{mg} \mathrm{kg}^{-1}\right)$ & $96.0 \pm 1.7$ & $75.0 \pm 1.4$ & $65.0 \pm 1.9$ & $115.0 \pm 1.5$ \\
$\mathrm{Ni}\left(\mathrm{mg} \mathrm{kg}^{-1}\right)$ & $25.0 \pm 1.3$ & $25.0 \pm 1.7$ & $17.0 \pm 1$ & $82.0 \pm 1.1$ \\
$\mathrm{~Pb}\left(\mathrm{mg} \mathrm{kg}^{-1}\right)$ & $18.0 \pm 0.5$ & $54.0 \pm 0.8$ & $31.0 \pm 0.1$ & $99.0 \pm 0.5$ \\
\hline
\end{tabular}

media \pm desviación estándar. 
los resultados presentados por Alvarenga et al. (2015) siendo de $5.4 \pm 0.5$ para LR.

El metal pesado que mayor contenido presentó en los residuos orgánicos, incluyendo el suelo, fue el $\mathrm{Cu}$ y presentó los valores más altos en el LR $(490 \pm 9.9$ $\mathrm{mg} \mathrm{kg}^{-1}$ ), pero al comparar este mismo metal en EB Garg y Gupta (2011) señalan un valor mayor ( $87 \pm 5.4$ $\mathrm{mg} \mathrm{kg}{ }^{-1}$ ) que el determinado en este trabajo.

Los valores de $\mathrm{Zn}$ y $\mathrm{Ni}$ en $\mathrm{EB}$ presentaron un valor más bajo que el reportado por Kharrazi et al. (2014); ellos encontraron para Zn $150 \pm 11.6 \mathrm{mg} \mathrm{kg}^{-1}$ y para Ni $41.16 \pm 5.15 \mathrm{mg} \mathrm{kg}^{-1}$ trabajando con estiércol bovino, con el propósito de hacer composta y vermicomposta.

El Zn determinado para RD se encontró en menor cantidad que el Zn (204 $\left.\pm 1.27 \mathrm{mg} \mathrm{kg}^{-1}\right)$ reportado por Barrena et al. (2014) que compararon residuos domésticos contra residuos de tipo industrial.

Estudios de Romero et al. (2010) que también determinaron $\mathrm{Zn}\left(151 \pm 3 \mathrm{mg} \mathrm{kg}^{-1}\right)$ en residuos de tipo agroindustrial fueron similares a los $\mathrm{RD}$ reportados en este estudio para el vermicomposteo.

Trabajos de Suthar (2009) y Silva et al. (2014) respectivamente, encontraron valores en LR entre 141.67 y $321.7 \mathrm{mg} \mathrm{kg}^{-1}$ de $\mathrm{Zn}$ que rebasan el contenido de Zn que se determinó en los LR de este trabajo.

Con respecto al $\mathrm{Pb}$ en $\mathrm{LR}$, estudios de Ouni et al.
(2013) encontraron un contenido $\left(254 \pm 5.15 \mathrm{mg} \mathrm{kg}^{-1}\right)$ que fue mayor al del LR de este experimento; cabe resaltar que durante el vermicomposteo la lombriz de tierra tiene una afinidad por acumular $\mathrm{Pb}$ y $\mathrm{Cd}$ (Mohee y Soobhan 2014).

De esta manera se puede decir que el contenido de metales pesados en el suelo, RD, EB y LR no rebasaron los límites permisibles de la norma NOM004-2002 (SEMARNAT 2002) donde la norma señala como límites máximos permisibles son; para $\mathrm{Cu}\left(1500 \mathrm{mg} \mathrm{kg}^{-1}\right), \mathrm{Zn}\left(2800 \mathrm{mg} \mathrm{kg}^{-1}\right)$, Ni (420 mg $\left.\mathrm{kg}^{-1}\right)$ y $\mathrm{Pb}\left(300 \mathrm{mg} \mathrm{kg}^{-1}\right)$ lo que revela su potencial uso agrícola. Además estudios de Mohee y Soobhan (2014) mencionan que metales como $\mathrm{Cd}, \mathrm{Co}, \mathrm{Mn}, \mathrm{Ni}$, $\mathrm{Pb}$ y $\mathrm{Zn}$ en LR sometidos a vermicomposteo disminuyen en su contenido; por ejemplo $\mathrm{Cu}$ disminuye entre 17 y $33 \%$, Cr de 10 a $50 \%$, Ni entre 8 y $48 \%$, Pb de 31 a $56 \%$ y $\mathrm{Zn}$ se ha visto que disminuye entre 11 a $36 \%$, por lo que al decrecer su contenido se pueden utilizar en suelos agrícolas.

El uso de residuos orgánicos no convencionales como son los LR de tipo municipal al ser sometidos a vermicomposteo van a incrementar la disponibilidad de nutrientes y a su vez reducir la disponibilidad de metales pesados en el sustrato, por el papel que desempeña la lombriz de tierra al bioacumular metales pesados (Mohee y Soobhan 2014).
(B)

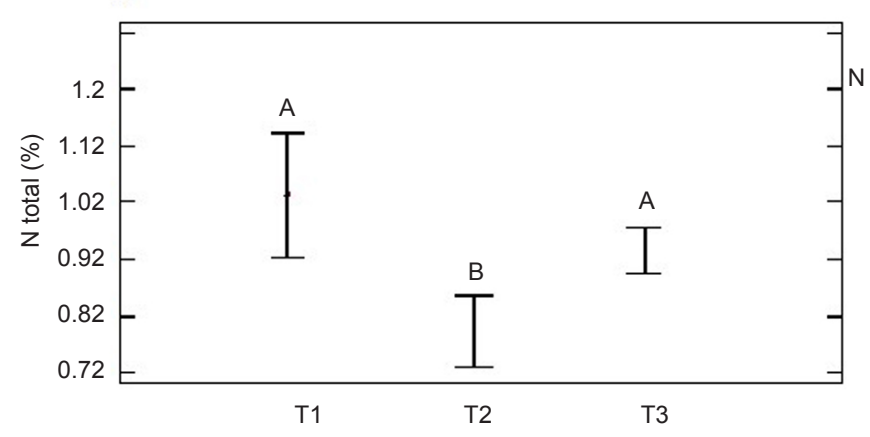

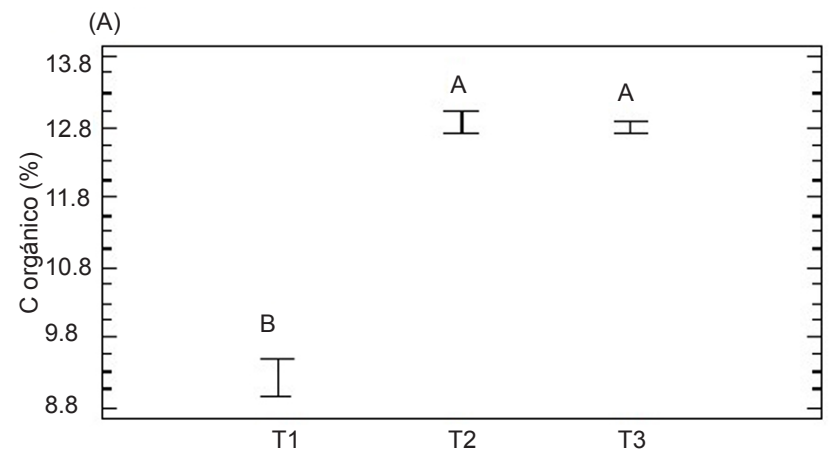

(C)

Fig. 1. Contenido de $\mathrm{C}$ orgánico (A), $\mathrm{N}$ total (B) y relación $\mathrm{C} / \mathrm{N}(\mathrm{C})$ en la vermicomposta de tres tratamientos, T1: estiércol de vaca y residuo vegetal (1:1), T2: estiércol de vaca y residuos vegetal (3:1) y T3: estiércol de vaca (100\%) donde cada tratamiento contiene $40 \mathrm{Mg} \mathrm{ha}^{-1}$ de LR 


\section{Vermicomposta}

El porcentaje de humedad fue similar en los tres tratamientos con un promedio de $21.63 \pm 0.65$ que se encuentra dentro del 20 y $40 \%$ recomendado por la norma oficial mexicana para el humus de lombriz (NMX-FF-109-SCFI-2007). Los valores de $\mathrm{pH}$ para $\mathrm{T} 1, \mathrm{~T} 2$ y $\mathrm{T} 3$ presentaron un comportamiento ligeramente alcalino $(7.93 \pm 0.09)$ el cual entra dentro del rango de la norma mexicana NMXFF-109-SCFI-2007 que presenta valores de $\mathrm{pH}$ entre 6.5 y 8.5 , el valor de $\mathrm{pH}$ medido permitió un buen crecimiento y desarrollo de la lombriz y está de acuerdo a lo reportado por Negi y Suthar (2013) los cuales mencionan que un incremento del $\mathrm{pH}$ se debe a una degradación de cadenas cortas de ácidos grasos y humus enlazados a cationes libres que están presentes en vermicomposteo. Singh et al. (2005), encontraron que las lombrices son muy sensibles a cambios de $\mathrm{pH}$, por lo que en el suelo se convierte en un factor que limita la distribución y el número de especies.

Con respecto al contenido de $\mathrm{C}_{\text {org }}$ (Fig. 1A) este presentó diferencias significativas $(\mathrm{p}<0.001)$ donde T2 y T3 presentaron valores muy similares $(12.88$ y 12.84 ) y diferentes a $\mathrm{T} 1$ (8.9), estos valores de $\mathrm{C}_{\text {org }}$ al transformarse a valores de MO, solo T2 y T3 (22.20, $22.13 \%)$ demostraron estar dentro de lo valores (20 y $50 \%$ ) recomendados por la norma NMX-FF-109SCFI-2007. Los cambios en $\mathrm{C}_{\text {org }}$ se deben a que la lombriz de tierra y los microorganismos llevan a cabo un consumo de este transformándolo en $\mathrm{CO}_{2}$ durante la actividad respiratoria, favoreciendo así la mineralización de la MO (Hiat y Tare 2011) y la formación de una fracción húmica madura que le va a dar al suelo una estabilidad física y aporte de nutrientes a la planta (Suthar y Singh 2008, Ngo et al. 2012, Zhang et al. 2012).

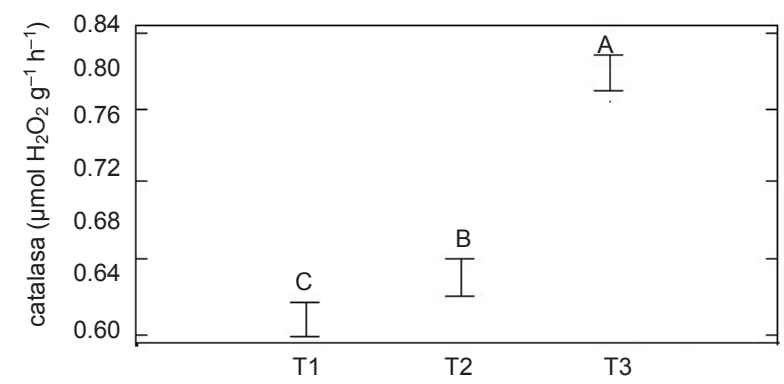

Fig. 2. Contenido de catalasa ( $\mu$ mol $\left.\mathrm{H}_{2} \mathrm{O}_{2} \mathrm{~g}^{-1} \mathrm{~h}^{-1}\right)$ durante el proceso de vermicomposteo en los tres tratamientos T1: estiércol de vaca y residuo vegetal 1:1), T2: estiércol de vaca y residuos vegetal $(3: 1)$ y T3: estiércol de vaca $(100 \%)$. Cada tratamiento contiene $40 \mathrm{Mg}$ ha-1 de LR
En todos los tratamientos el porcentaje de $\mathrm{N}$ total (Fig. 1B) se encontró dentro de los valores reportados por la NMX-FF-109-SCFI-2007 (< $4 \%)$; T1 $(1.03 \pm 0.17)$ seguido por $\mathrm{T} 3(0.98 \pm 0.03 \%)$ y T2 $(0.73 \pm 0.02 \%)$.

El contenido de $\mathrm{N}$ total que se generó en el proceso se debió a la elaboración de productos (metabolitos) ricos en $\mathrm{N}$, también al $\mathrm{N}$ producido por la excreta, el moco y los fluidos que la lombriz elimina. Por otra parte las enzimas facilitan la formación de compuestos como el $\mathrm{NH}_{4}{ }^{+}$, aunado a esto en la lombriz está presente el $\mathrm{N}$ en el tejido muerto y también al $\mathrm{N}$ producido durante el proceso de mineralización durante el vermicomposteo (Gupta y Garg 2008).

La relación $\mathrm{C} / \mathrm{N}$ aunque fue diferente entre los tres tratamientos (Fig. 1C), esta fue adecuada para los tratamientos, en estos, se presenta un grado de estabilización y madurez, siendo los valores para T3 y T2 $(14.14 \pm 0.48$ y $17.48 \pm 0.64)$ mientras que para T1 (8.78 \pm 1.48$)$ presentó el valor más bajo.

Los productos derivados de la vermicomposta con una relación $\mathrm{C} / \mathrm{N}$ entre 20 y 12 se consideran adecuados para uso forestal y por debajo de $10 \mathrm{se}$ valoran aptos para uso agrícola (Hiat y Tare 2011). Por lo que el T1 se puede usar como un producto para uso agronómico, por otra parte la relación $\mathrm{C} / \mathrm{N}$ determina la edad de la lombriz; un valor menor a 20 presenta organismos maduros $(60 \%)$ y valores mayores a 20 predominan juveniles $(70 \%)$ durante el periodo de incubación (Garg et al. 2006).

La actividad enzimática de la catalasa presentó diferencias $(\mathrm{p}<0.001)$ donde el tratamiento que responde de manera positiva a la catalasa es T3 $\left(0.81 \pm 0.015 \mu \mathrm{mol} \mathrm{H} 2 \mathrm{O} 2 \mathrm{~g}^{-1} \mathrm{~h}^{-1}\right)$ seguido de T2 y T1 $\left(0.64 \pm 0.015\right.$ y $\left.0.61 \pm 0.015 \mu \mathrm{mol} \mathrm{H} 2 \mathrm{O} 2 \mathrm{~g}^{-1} \mathrm{~h}^{-1}\right)$ respectivamente (Fig. 2). La actividad de la catalasa se incrementó solo para el tratamiento que contenía estiércol bovino (T3), el T1 y T2 presentaron valores bajos de actividad enzimática.

Es importante mencionar que una mayor actividad de la catalasa favorece a que los microorganismos lleven adecuadamente sus actividades metabólicas, ya que funciona como agente antioxidante $\mathrm{y}$ al estar presente en la vermicomposta y en el suelo, la planta resulta beneficiada en su fisiología (Xue y Heang 2013).

La actividad de la catalasa se ve respaldada cuando el residuo orgánico está estabilizado por la técnica del vermicomposteo (Trasar-Cepeda et al. 2003) además un incremento de la biomasa microbiana favorece la degradación de la $\mathrm{MO}$ y el incremento de la actividad de la enzima, lo que se afectó por la presencia del metal pesado en los tratamientos que contenían LR, caso similar se presentó en estudios 
de Ouni et al. (2013) donde el incremento de la MO ayuda a aumentar la actividad enzimática favoreciendo la degradación de la MO.

\section{CONCLUSIONES}

La presencia del lodo residual y la misma proporción de estiércol bovino y residuos domésticos en la elaboración de la vermicomposta potencializa la estabilidad de la materia orgánica y llega a una madurez en el producto elaborado.

El contenido de metales pesados presentes en el suelo, RD, EB y LR no rebasaron los límites permisibles de las normas NOM-021-2000 (SEMARNAT 2000) y la NOM-004-2002 (SEMARNAT 2002).

El C orgánico en los tratamientos estuvo de acuerdo con la norma NMX-FF-109-SCFI-2007. El mayor contenido de $\mathrm{N}$ se dio en $\mathrm{T} 1$ con respecto a los otros tratamientos. La relación C/N $(8.64 \pm 1.48)$ en T1 fue la más adecuada y estable por lo que este tratamiento se puede utilizar en uso agronómico.

La dinámica que se dio en el proceso es mostrada por la enzima catalasa que demostró ser un indicador bioquímico que determina la calidad del producto elaborado, siendo T3 el de mayor actividad.

Considerando al $\mathrm{N}$ total y la relación $\mathrm{C} / \mathrm{N}$ como indicadores de estabilidad se concluye que $\mathrm{T} 1$ fue la mejor opción para su uso agronómico.

\section{AGRADECIMIENTOS}

Los autores agradecen a Rosaura Miranda V. por el trabajo de laboratorio desarrollado.

\section{REFERENCIAS}

Alvarenga P., Mourinha C. y Farto M., Santos T., palma P., Sengo J., Morais M.C. y Queda C.C. (2015). Sewage sludge compost and other representative organic waste as agricultural soil amendments: Benefits versus limiting factor. Waste Manage. 40, 44-52. http://dx.doi.org/10.1016/j. wasman.2015.01.027

Armijo V., Ojeda S. y Ramírez M.E. (2007). Caracterización de residuos sólidos el potencial de reciclaje para una institución de educación superior. Memorias. Encuentro Nacional de Expertos en Residuos Sólidos. Universidad Autónoma de Baja California. México. ISBN 970-735-075-X: pp. 66-77.
Awasthi M.K., Pandey A.K., Bundela P.S. y Khan J. (2015). Co-composting of organic fraction of municipal solid waste mixed with different bulking waste: Characterization of physichemical paramenters and microbial enzymatic dynamic. Biores. Technol. 182, 200-207. http://dx.doi. org/10.1016/j.biortech.2015.01.104

Barrena R., Font X., Gabarrell X. y Sánchez A. (2014). Home composting versus industrial composting: Influence of composting system on compost quality with focus compost stability. Waste Manage. 34, 1109-1116. http://dx.doi. org/10.1016/j.wasman.2014.02.008

Bremner J.M. y Mulvaney R.G. (1982). Nitrogen total, in: Page A.L., Miller R.H., Keeney D.R. (Eds). Methods of soil Analysis. Am. Soc. Agronomy, Madison Wisconsin, pp. 575-624.

Campitelli P. y Ceppi S. (2008). Chemical, physical and biological compost and vermicompost characterization: A chemometric study. Chemometr. Intell. Lab. 90, 64-71. http://dx.doi.org/10.1016/j. chemolab.2007.08.001

Ceron R. L. E. y Melgarejo M. L. M. (2005). Enzimas del suelo: indicadores de salud y calidad. Acta Biol. Ven. 10, 5-18.

Garg V.K., Gupta R. y Satya S. (2006). Vermicomposting of different types of waste using Eisenia foetida: a comparative study. Biores. Technol. 97, 391-395. http://dx.doi.org/10.1016/j.biortech.2005.03.009

Garg V.K. y Gupta R. (2011).Optimization of cow dung spiked pre-consumer processing vegetable waste for vermicomposting using Eisenia fetida. Ecotox. Environ. Safe. 74, 19-24. http://dx.doi. org/10.1016/j.ecoenv.2010.09.015

Gupta R. y Garg V.K. (2008). Stabilization of primary sewage sludge during vermicomposting. J. Hazar. Mater. 153, 1023-1030. http://dx.doi. org/10.1016/j.jhazmat.2007.09.055

Hernández-Rodríguez O.A., López-Díaz J.C., ArrasVota A.M., Quezada-Solis J. y Ojeda-Barrios D. (2012). Quality of vermicompost obtained from residues of forestry a livestock. Sustain. Agr. Res. 1, 70-76.

Hiat S. y Tare V. (2011). Optimizing vermistabilization of waste activated sludge using vermicompost as bulking material. Waste Manage. Res. 31, 502-511. http://dx.doi.org/10.1016/j. wasman.2010.11.004

Kharrazi S.M., Younesi H. y Torghabeh J.A. (2014). Microbial biodegradation of waste materials for nutrients enrichment and heavy metals removal: 
An integrated composting-vermicomposting process. Int. Biodeter. Biodegr. 92,41-48. http:// dx.doi.org/10.1016/j.ibiod.2014.04.011

Mohee R. y Soobhany N. (2014). Comparison of heavy metals content in compost against vermicompost of organic solid waste: Past and Present. Resour. Conserv. Recy.92, 206-213. http://dx.doi. org/10.1016/j.resconrec.2014.07.004

Negi R. y Suthar S. (2013). Vermistabilization of paper mill wastewater sludge using Eisenia fetida. Biores. Technol. 128, 193-198. http://dx.doi. org/10.1016/j.biortech.2012.10.022

Nelson D. W. y Sonmers L.F. (1996). Total carbon and organic carbon and organic matter. In: Page, A.L., Miller R.H., Keeney Society of Agronomy, Madison. USA. 539-579 pp.

NMX-FF-109-SCF-2007. Humus de Lombriz (Lombricomposta) Especificaciones y Métodos de prueba. 24 pags.

Ngo P.T., Rumpel C., Doam T. T. y Jouquet P. (2012). The effect of earthworms on carbon storage and soil organic matter composition in tropical soil amended with compost and vermicompost. Soil Biol. \& Biochem. 50, 214-220. http://dx.doi. org/10.1016/j.soilbio.2012.02.037

Ouni Y., Lakhar A., Scelza R., Scotti R., Abdelly C., Barboun Z. y Rao M.A., (2013). Effects of two composts and two grasses on microbial biomass and biological activity in a salt-affected soil. Ecol. Eng. 60,363-369. http://dx.doi.org/10.1016/j. ecoleng.2013.09.002

Pearson M.S., Maenpa K., Pierzynski G.M. y Lydy M.J. (2000). Effects of Soil Amendments on the Bioavailability of Lead, Zinc, and Cadmium to Earthworms Bioremediation and Biodegradation. J. Environ. Qual. 29, 161-1617. DOI:10.2134/jeq2000.00472425002900050031x Romero E., Bayo J.F., Díaz C.J.M. y Nogales R. (2010). Enzyme activities and diuron persistence in soil amended with vermicompost derived from spent grape marc and treated with urea. Appl. Soil Ecol. 44,198-204. http://dx.doi.org/10.1016/j. apsoil.2009.12.006

SEMARNAT (2000). Norma Oficial Mexicana NOM-021-2000 que establece las especificaciones de fertilidad, salinidad, y clasificación de suelos, estudios, muestreo y análisis. Diario Oficial, martes 31 de diciembre, 2002.

SEMARNAT (2002). Norma Oficial Mexicana NOM-004, Protección ambiental. - lodos y biosólidos- Especificaciones y límites máximos permisibles de contaminantes para su aprovechamiento y disposición oficial. Diario Oficial viernes 15 de agosto de 2003.

Silva M.D.M., Aceves B.M., Araújo A.S.F., Araíjo F.F. y Melo J. (2014). Soil Microbial Biomass three-year consecutive composted tannery sludge amendment. Pedosphere. 24,469-475. DOI: 10.1016/S1002-0160(14)60033-3

Singh N.B., Khare A.H., Bhargava D.S., Bhattacharya S. (2005). Effects of initial substrate $\mathrm{pH}$ on vermicomposting using Perionyx excavate (Perrier, 1872). Appl. Ecol. Environ. Res. 1, 85-97

Suthar S. y Singh S. (2008). Vermicomposting of domestic waste by using two epigeic earthworms (Perionyx escavatus and Perionyx sansibarius). Int. J. Environ. Sci. Technol. 1, 99-106. DOI: 10.1007/BF03326002

Suthar S. (2009). Vermistabilization of municipal sewage amended with sugarcane trash using epigeic Eisenia fetida (Oligochaeta). J. Hazar. Mater. 163, 199-206. http://dx.doi.org/10.1016/j. jhazmat.2008.06.106

Trasar-Cepeda C., Gil-Sotres F. y Leiros M.C., (2003). Determinación de la actividad catalasa del suelo. En: Técnicas de análisis de parámetros bioquímicos en suelos. (García I.C., Gil S.F., Hernández F.T., Trasar C.C. eds) Mundi Prensa. Madrid, España. pp 229-244.

Xue D. y Heang X. (2013). The impact of sewage sludge compost on tree peony growth and soil microbiological, and biochemical properties. Chemosphere. 93, 583-589. http://dx.doi. org/10.1016/j.chemosphere.2013.05.065

Zhang Z., Chen Q., Yin C., Shen X., Chen X. y Sun H. (2012). The effects of organics matter on the physiological features of Malus hupehensis seeldings and soil properties under replant conditions. Sci. Hort. 146, 52-58. http://dx.doi.org/10.1016/j. scienta.2012.08.011 